\title{
Análise da variabilidade pluviométrica utilizando a frequência de ocorrência e as medidas de assimetria e curtose na bacia hidrográfica do Rio Araguari - (MG), período de 1975 a 2014
}

\author{
Samuel Alves Maciel ${ }^{(\mathrm{a})}$, Luiz Antônio de Oliveira ${ }^{(\mathrm{b})}$ \\ (a) Instituto de Geografia, Universidade Federal de Uberlândia, samuel_alves@ufu.br \\ (b) Instituto de Geografia, Universidade Federal de Uberlândia, luizantonio@ig.ufu.br
}

Eixo: Climatologia em difentes níveis escalares: mudanças e variabilidades

Resumo

\begin{abstract}
Este trabalho tem como objetivo analisar a variabilidade pluviométrica, com base na frequência de ocorrência e nas medidas de Assimetria e Curtose, da bacia hidrográfica do Rio Araguari - (MG), no período de 1975 a 2014. Foram utilizados dados de precipitação diária $(\mathrm{mm})$, de 12 postos pluviométricos localizados ao longo da bacia, série histórica de 40 anos. Após a tabulação para determinar as precipitações acumuladas mensalmente, seguiram-se as etapas metodológicas do computo da amplitude da variação, número e amplitude do intervalo de classes, frequência de ocorrência simples e coeficientes de Assimetria e Curtose. A partir dos resultados inferiu-se que dos 12 postos selecionados na área de estudo, 33,3\% ou 4 deles foram classificados como Distribuição Assimétrica Positiva Moderada e 66,7\% ou 8 deles foram categorizados como Distribuição Assimétrica Positiva Forte. Em termos de percentagens ainda, 25\% ou 3 deles apresentaram curva Leptocúrtica e $75 \%$ ou 9 deles expressaram curva Platicúrtica.
\end{abstract}

Palavras chave: Variabilidade Pluviométrica, Frequência de Ocorrência, Assimetria e Curtose, Bacia Hidrográfica do Rio Araguari.

\section{Introdução}

A precipitação é um dos principais elementos físicos pertinentes a classificação climática de uma determinada área, bem como, é base para os processos de gestão ambiental, econômico e social. Dessa forma, faz-se necessário compreender a variabiliade pluviométrica espacial e temporal, procurando assim, planejar e organizar os diversos setores da sociedade influenciados por esta variável, como a produção agrícola, a contrução civil, a geração de energia elétrica, o abastecimento urbano, o planejamento de recursos hídricos regionais e municipais, e demais.

De modo geral, a precipitação é caracterizada como toda forma de água oriunda da atmosfera e que atinge a superfície terrestre como chuva, granizo, orvalho, neblina, neve ou geada. Sua formação está associada à ascensão das massas de ar úmidas, devida aos seguintes fatores: convecção térmica, relevo e ação frontal das massas. Tal ascensão do ar ocasiona um resfriamento que pode fazê-lo alcançar o seu ponto de saturação, tendo em seguida a condensação do vapor de água na forma de minúsculas gotas que são mantidas em suspensão, sob nuvens e nevoeiros. Para que ocorra um evento de precipitação é preciso que estas gotas aumentem a partir de núcleos de condensação, como por 
exemplo, gelo, poeira ou demais partículas, até que atinjam o peso necessário para superarem as forças de sustentação e caírem. (HOLTZ, 1976).

Segundo Ayoade (1996) ocorre uma relação inversa entre a quantidade de precipitação pluvial e sua variabilidade. Uma vez que, a precipitação é mais variável nas áreas secas e subúmidas e a variabilidade da precipitação pluvial é pequena na regiões úmidas dos trópicos e nas latitudes médias ciclônicas. $\mathrm{O}$ autor, ainda afirma que as consequências econômicas da variabilidade da precipitação pluvial são, todavia, mais severas em áreas agrícolas intensamente povoadas das regiões úmidas do que nas regiões frias ou secas, esparsamente povoadas.

Para Christofoletti (2000) a variabilidade climática pode ser entendida como a maneira pela qual se comporta a variação de valores de um elemento climático dentro de um determinado período de registros. Assim, neste trabalho optou-se em compreender a variabilidade das chuvas por meio da utlização da frequência de ocorrência e das medidas de assimetria e curtose para as séries de dados utilizadas. Neste caso, o grau em que os valores de x se dispersam em torno da média é dado pelo parâmetro denominado escala. Já o valor em torno do qual os valores de x se dispersam é denominado parâmetro de posição. E por último, o parâmetro destinado a forma das séries está associado à assimetria das frequências. (GOMES, 2011)

Diversos autores como Vieira, Camargo e Siqueira (2009), Sccol, Cardoso e Miquelluti (2010), Silva, Lima e Bottega (2011) e Mello e Oliveria (2016) realizaram suas pesquisas analisando o comportamento das séries históricas de chuvas a partir dos valores gerados pelos coeficientes de assimetria e curtose.

Assim, a área de estudo deste trabalho compreende a Bacia Hidrográfica do Rio Araguari (BHRA), figura 1, situada na Mesorregião do Triângulo Mineiro e Alto Paranaíba, na porção oeste do estado de Minas Gerais, compreendendo 20 municípios, parcial ou totalmente, com área de $22.186 \mathrm{~km}^{2}$. O rio Araguari é afluente da margem esquerda do Rio Paranaíba e nasce no Parque Nacional da Serra da Canastra, localizado no município de São Roque de Minas, percorrendo $745 \mathrm{~km}$ até sua foz no Rio Paranaíba. 


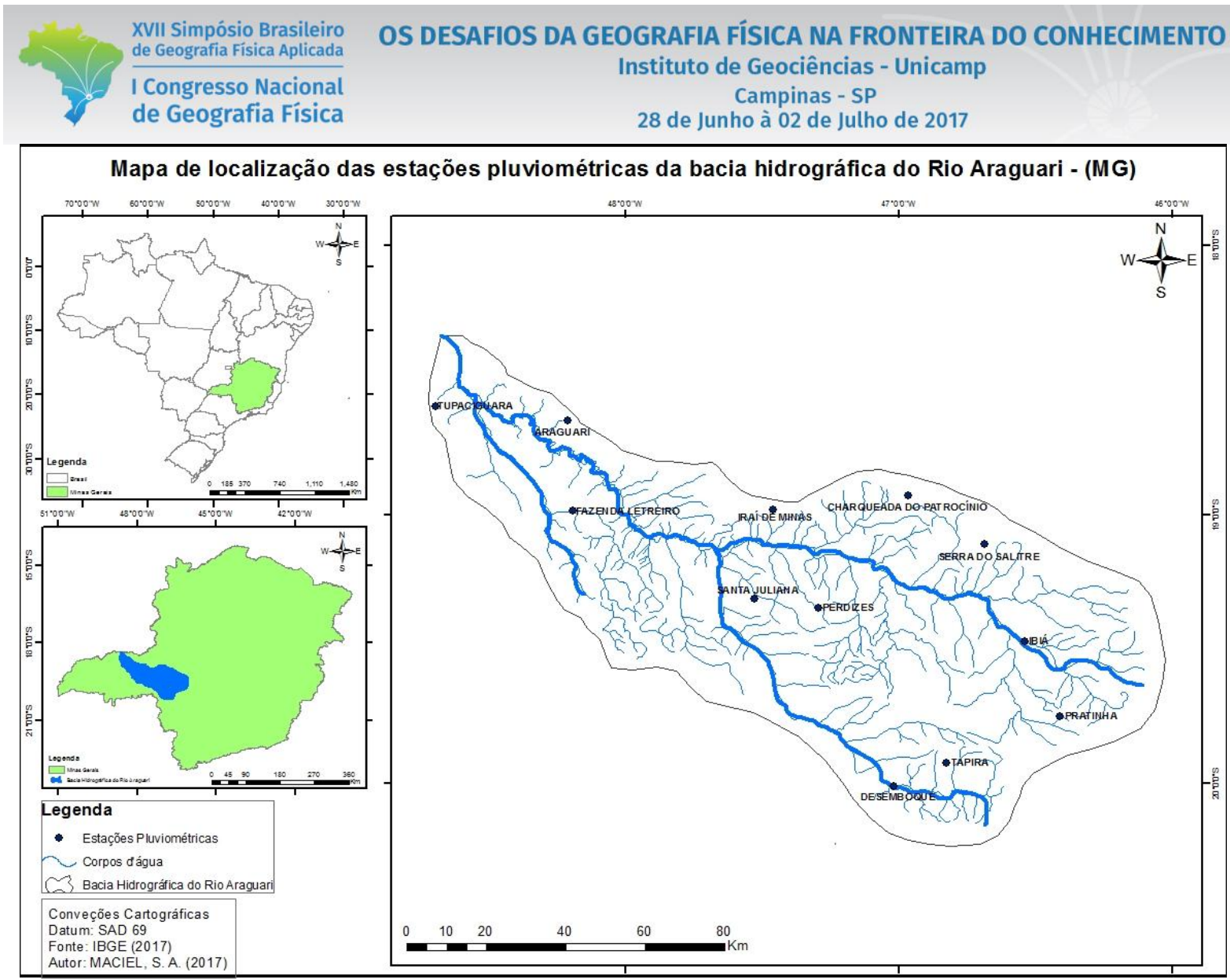

Figura 1 - Mapa de localização da bacia hidrográfica do Rio Araguari - (MG) e do posicionamento das estações pluviométricas.

Fonte dos dados: IBGE (2017).

Organização: MACIEL, S. A. (2017).

De acordo com os pressupostos teóricos e a relevância da temática abordada, este estudo tem como objetivo analisar a variabilidade pluviométrica, com base na frequência de ocorrência e nas medidas de assimetria e curtose, da bacia hidrográfica do Rio Araguari - (MG), durante o período de 1975 a 2014.

\section{Materiais e Métodos}

Para elaboração deste trabalho inicialmente foi construído um referencial teórico - conceitual acerca do tema estudado. $\mathrm{Na}$ fase de processamento foram utilizados dados de precipitação diária $(\mathrm{mm})$, período de 24 horas, de 12 estações pluviométricas localizadas ao longo da bacia hidrográfica do Rio Araguari, conforme a tabela I. Estes constituíram uma série histórica de 40 anos, entre 1975 a 2014. Neste período, em função da presença de falhas nas séries de dados alguns anos foram eliminados, o que por sua vez, não prejudicou os resultados da pesquisa. Os dados foram disponibilizados pelo Sistema de Informações Hidrológicas (HidroWeb) de responsabilidade da Agência Nacional de Águas (ANA).

Tabela I - Localização das estações pluviométricas na bacia hidrográfica do Rio Araguari - (MG).

\begin{tabular}{|c|c|c|c|c|c|c|c|}
\hline $\mathbf{N}^{\circ}$ & Código & $\begin{array}{c}\text { Nome } \\
\text { do Posto }\end{array}$ & Município de Instalação & $\begin{array}{l}\text { Latitude } \\
\text { (S) }\end{array}$ & $\begin{array}{l}\text { Longitude } \\
\text { (W) }\end{array}$ & $\begin{array}{l}\text { Altitude } \\
\text { (m) }\end{array}$ & $\begin{array}{l}\text { Falhas } \\
\text { (anos) }\end{array}$ \\
\hline 1 & 1848010 & Araguari & Araguari & $18^{\circ} 39^{\prime} 04^{\prime \prime}$ & $48^{\circ} 12^{\prime} 33^{\prime \prime}$ & 963 & 2 \\
\hline 2 & 1946004 & Ibiá & Ibiá & $19^{\circ} 28^{\prime} 30^{\prime \prime}$ & $46^{\circ} 32 ' 31^{\prime \prime}$ & 855 & - \\
\hline 3 & 1847010 & Iraí de Minas & Iraí de Minas & $18^{\circ} 58 ' 55^{\prime \prime}$ & $47^{\circ} 27^{\prime} 27^{\prime \prime}$ & 946 & 1 \\
\hline
\end{tabular}


XVII Simpósio Brasileiro

de Geografia Fisica Aplicada

I Congresso Nacional

de Geografia Física

\section{OS DESAFIOS DA GEOGRAFIA FÍSICA NA FRONTEIRA DO CONHECIMENTO \\ Instituto de Geociências - Unicamp \\ Campinas - SP \\ 28 de Junho à 02 de Julho de 2017}

1846002 Charqueada de Patrocínio

Perdizes

Pratinha

Desemboque

Santa Juliana

Serra do Salitre

Tapira

Fazenda Cachoeira

Fazenda Letreiro $19^{\circ} 20^{\prime} 55^{\prime \prime} \quad 47^{\circ} 17^{\prime} 43^{\prime \prime}$

$19^{\circ} 45^{\prime} 05^{\prime \prime} \quad 46^{\circ} 24 ' 43^{\prime \prime}$

$20^{\circ} 00^{\prime} 49^{\prime \prime} \quad 47^{\circ} 01^{\prime} 09^{\prime \prime}$ $18^{\circ} 55^{\prime} 48^{\prime \prime} \quad 46^{\circ} 58^{\prime} 00^{\prime \prime}$ $\begin{array}{ccccc}\text { Santa Juliana } & 1^{\circ} 18^{\prime} 57^{\prime \prime} & 47^{\circ} 31^{\prime} 34^{\prime \prime} & 950 & 1 \\ \text { Serra do Salitre } & 1^{\circ} 06^{\prime} 46^{\prime \prime} & 46^{\circ} 41^{\prime} 18^{\prime \prime} & - & 3\end{array}$

$\begin{array}{ccccc}\text { Santa Juliana } & 1^{\circ} 18^{\prime} 57^{\prime \prime} & 47^{\circ} 31^{\prime} 34^{\prime \prime} & 950 & 1 \\ \text { Serra do Salitre } & 1^{\circ} 06^{\prime} 46^{\prime \prime} & 46^{\circ} 41^{\prime} 18^{\prime \prime} & - & 3\end{array}$

Tapira $\quad 1^{\circ} 55^{\prime} 37^{\prime \prime} 46^{\circ} 49^{\prime} 31^{\prime \prime} \quad-\quad 18$

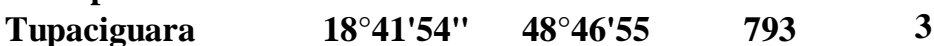

Uberlândia $\quad 18^{\circ} 59^{\prime} 18^{\prime \prime} \quad 48^{\circ} 11 ' 25^{\prime \prime}$

960

789

$-$

960

$\begin{array}{ccccc}\text { Santa Juliana } & 1^{\circ} 18^{\prime} 57^{\prime \prime} & 47^{\circ} 31^{\prime} 34^{\prime \prime} & 950 & 1 \\ \text { Serra do Salitre } & 1^{\circ} 06^{\prime} 46^{\prime \prime} & 46^{\circ} 41^{\prime} 18^{\prime \prime} & - & 3\end{array}$

$\begin{array}{ccccc}\text { Santa Juliana } & 1^{\circ} 18^{\prime} 57^{\prime \prime} & 47^{\circ} 31^{\prime} 34^{\prime \prime} & 950 & 1 \\ \text { Serra do Salitre } & 1^{\circ} 06^{\prime} 46^{\prime \prime} & 46^{\circ} 41^{\prime} 18^{\prime \prime} & - & 3\end{array}$

Fonte: HIDROWEB/ANA (2017). Organização: MACIEL, S. A. (2017).

Os valores das precipitações mensais acumuladas ao longo de toda a série histórica que posteriormente foram utilizados na aplicação da frequência de ocorrência e nas medidas de assimetria e curtose, foram executados com auxílio do software computacional Sistema de Informações Hidrológicas, versão 1.2 (HIDRO 1.2).

Inicialmente para o estabelecimento das frequências absolutas de cada posto selecionado na bacia, já com o número de elementos da amostra (n) encontrado, determinou-se, então, a amplitude da variação das precipitações pertinentes as séries históricas, como especificado na equação 01 :

$$
\text { A= Pmáx - Pmín Equação } 01
$$

Onde: $\mathrm{A}$ = amplitude dos dados de precipitação; Pmáx = Maior valor de precipitação; e Pmín = Menor valor de precipitação.

Assim, pode-se estabelecer o número de intervalo de classes $(\mathrm{N})$, verificado na equação 02 :

$$
\mathrm{N}=1+3,3 * \log \mathrm{n} \text { Equação } 02
$$

Onde: $\mathrm{N}=$ número de intervalos de classes e $\mathrm{n}=$ número de elementos da amostra de precipitações mensais acumuladas.

Para realização da distribuição dos dados, de acordo com a amplitude total do intervalo de classes (K), utilizou-se a seguinte equação 03:

$$
\mathrm{K}=\frac{\mathrm{A}}{\mathrm{N}} \text { Equação } 03
$$

Onde: $\mathrm{K}=$ amplitude do intervalo de classes; $\mathrm{A}=$ amplitude dos dados de temperatura e $\mathrm{N}=$ número de intervalo de classes.

Com estas etapas realizadas, calculou-se a frequência de ocorrência simples das precipitações mensais acumuladas dentro de cada intervalo que posteriormente auxiliaram na determinação e análise dos resultados gerados a partir das medidas de distribuições assimétrica e curtose. 


\section{OS DESAFIOS DA GEOGRAFIA FÍSICA NA FRONTEIRA DO CONHECIMENTO \\ Instituto de Geociências - Unicamp \\ Campinas - SP \\ 28 de Junho à 02 de Julho de 2017}

$\mathrm{Na}$ análise da série temporal de precipitação mensal acumulada (1975-2014) de cada estação pluviométrica da bacia, verificou-se que os valores gerados de média geral, moda e mediana não foram coincidentes, fazendo com que as distribuições de dados fossem consideradas assimétricas.

Nesse sentido, as distribuições classificadas como assimétricas possuem uma espécie de "cauda" em uma de suas extremidades, sendo que, quando esta à direita, é positivamente assimétrica, e quando está a esquerda, é negativamente assimétrica. (MEDRI, 2011).

Dentre as fórmulas existentes para o cálculo do coeficiente de assimetria, optou-se por utilizar neste trabalho a referente ao Coeficiente de Pearson:

$$
A S=\frac{\bar{x}-\mathrm{x}_{\mathrm{mo}}}{\mathrm{S}} \text { Equação } 04
$$

Onde: $A S=$ coeficiente de variação; $\bar{x}=$ média da distribuição; $\mathrm{x}_{\mathrm{mo}}=$ moda da distribuição e $\mathrm{S}=$ desvio padrão da distribuição.

No caso de: $A S=0$, a distribuição é considerada simétrica; $A S>0$, a distribuição é assimétrica positiva ou à direita e $A S<0$, a distribuição é assimétrica negativa ou à esquerda.

Com os resultados encontrados a partir do Coeficiente de Assimetria de Pearson, pode-se assim, avaliar os graus de assimetria entre as distribuições dos postos, sendo que, quanto maior o AS, mais assimétrica é a curva.

Para intervalos em que: $0<\mathrm{AS}<0,15$. a Assimetria é fraca; $0,15<\mathrm{AS}<1$, a Assimetria é moderada e AS $\geq 1$, a Assimetria é forte.

Já, a medida de curtose pode ser definida como o grau de achatamento - ou afilamento - de uma distribuição em relação a uma distribuição padrão, denominada de curva normal padrão. A curva normal é caracterizada como a base referencial e é chamada de mesocúrtica. Quando a curva de frequência é mais achatada (afilada) do que a normal recebe a nomenclatura de leptocúrtica. E por fim, se a curva de frequência for mais aberta do que a normal, esta recebe o nome de platicúrtica. (MEDRI, 2011).

O cálculo utilizado para a determinação do Coeficiente de Curtose para a série temporal (1975 - 2014) de todos os postos foi:

$$
C=\frac{Q_{3}-Q_{1}}{2\left(\mathrm{P}_{90}-P_{10}\right)} \quad \text { Equação } 05
$$




\section{OS DESAFIOS DA GEOGRAFIA FÍSICA NA FRONTEIRA DO CONHECIMENTO \\ Instituto de Geociências - Unicamp \\ Campinas - SP \\ 28 de Junho à 02 de Julho de 2017}

Sendo que: $\mathrm{C}=$ coeficiente de curtose; $Q_{3}=$ terceiro quartil; $Q_{1=\text { primeiro quartil; }} P_{10}=$ décimo percentil e $P_{90}=$ nonagésimo percentil.

Para estabelecimento de comparações entre as diversas curvas e suas respectivas classificações, considerou-se que: $\mathrm{C}=0,263$ corresponde a curva mesocúrtica; $\mathrm{C}<0,263$ corresponde a curva leptocúrtica e C >0,263 corresponde a curva platicúrtica.

\section{Resultados}

O gráfico apresentado na figura 2 demonstra a sazonalidade marcante na bacia hidrográfica do Rio Araguari - MG, onde, no geral a estação chuvosa ocorre de outubro a março e o período de estiagem de abril a setembro.

Os maiores valores médios de precipitação mensal ocorrem em dezembro e janeiro, sendo 287,2 mm e 293,2 mm, respectivamente, e diminui de maneira progressiva até o mês de julho, média de 14,0 mm. O total anual acumulado médio de chuvas para a bacia é de $1.522,6 \mathrm{~mm}$. Sendo que, a estação chuvosa, concentra a maior parte da pluviosidade anual, com $84,7 \%$ das chuvas ou $1.289,7 \mathrm{~mm}$, enquanto que o período de estiagem representa $15,3 \%$ das chuvas anuais ou $232,9 \mathrm{~mm}$.

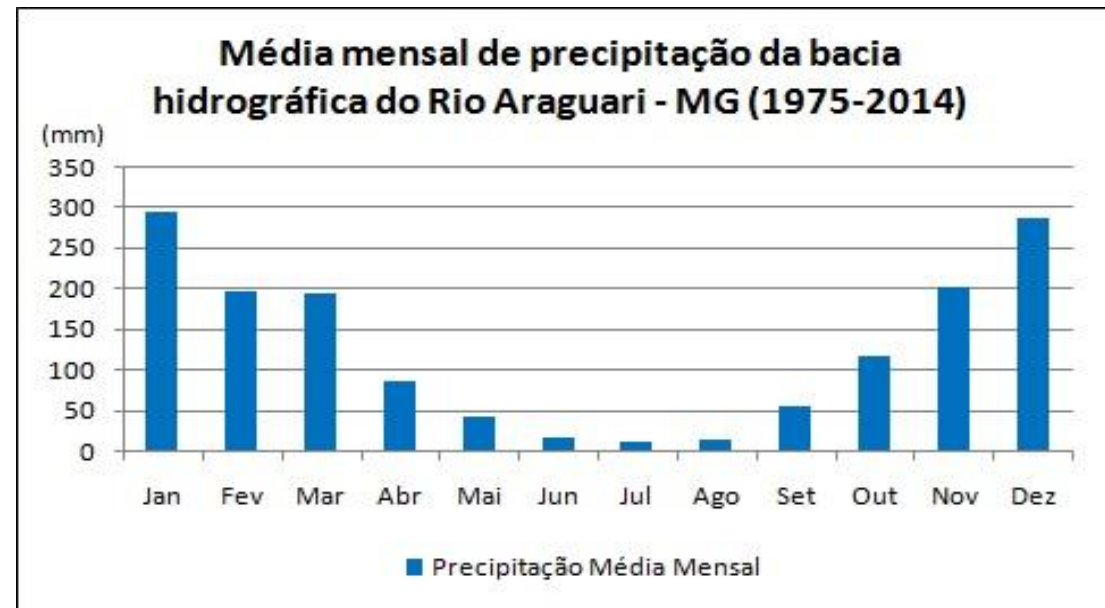

Figura 2 - Média das alturas pluviométricas mensais dos postos pluviométricos localizados na área de estudo.

Fonte dos dados: HidroWeb/ANA (2017).

Organização: MACIEL, S. A. (2017).

Os gráficos a seguir, ilustrados na figura 3 , corromboram com os resultados para posterior análise dos coeficientes de assimetria e curtose, a partir das informações referentes aos valores de frequência absoluta de cada posto pluviométrico selecionado na bacia (1975 - 2014). A título de exemplos, foram selecionados 4 postos pluviométricos - Araguari, Ibiá, Sacramento e Santa Juliana - localizados em cada extremo e no centro da bacia, para elucidar os seus respectivos comportamentos. 
Na estação de Araguari, no intervalo total de 455 meses de registro, as precipitações variaram, tendo maior acumulado de valores entre 33,5 mm a 201,1 mm, o que significou na série histórica 293 meses,o que compreende de dados da série. Por outro lado, em 107 meses, as precipitações concentraram-se no intervalo de $234,7 \mathrm{~mm}$ a $569,9 \mathrm{~mm}$. Apenas dois meses, apresentaram precipitações mensais acumuladas extremas iguais ou superiores a 737,5 mm.

Na estação de Ibiá, no intervalo total de 468 meses de registro, as precipitações mensais variaram na maioria do tempo entre $24,4 \mathrm{~mm}$ a $245,2 \mathrm{~mm}$, totalizando 350 meses nessas condições. No restante, em 80 meses, as chuvas mensais estiveram no intervalo de $269,8 \mathrm{~mm}$ a $490,5 \mathrm{~mm}$. Somente em três meses, as precipitações mensais acumuladas extremas atigiram ou ultrapassaram 515,0 mm.

Já na estação de Sacramento, de 478 meses da série histórica total, 411 meses, registraram valores acumulados de precipitações mensais dentro do intervalo de 30,2 $\mathrm{mm}$ a $361,9 \mathrm{~mm}$. Nos demais, 29 meses, as chuvas variaram de $392,1 \mathrm{~mm}$ a $573,0 \mathrm{~mm}$. Sendo que, dois meses, acumularam precipitações mensais acumuladas extremas dentro de $633,4 \mathrm{~mm}$ a $663,5 \mathrm{~mm}$.

Por último, na estação de Santa Juliana, do total de 467 meses de registro,em 393 meses, as precipitações tiveram um maior valor acumulado entre os valores de $29,2 \mathrm{~mm}$ a $321,4 \mathrm{~mm}$. E durante 32 meses, as chuvas mensais estiveram no intervalo de $350,6 \mathrm{~mm}$ a 526,0 $\mathrm{mm}$. Apenas um mês, registrou precipitação mensal acumulada extrema igual ou superior a $642,9 \mathrm{~mm}$.

Dentro do período de estiagem, entre junho a agosto, alguns destes meses não apresentaram ocorrências de chuvas, portanto, não totalizaram volumes de precipitações mensais. Em Araguari, Ibiá, Sacramento e Santa Juliana (53, 35, 36 e 41 meses) estiveram nesta condição.

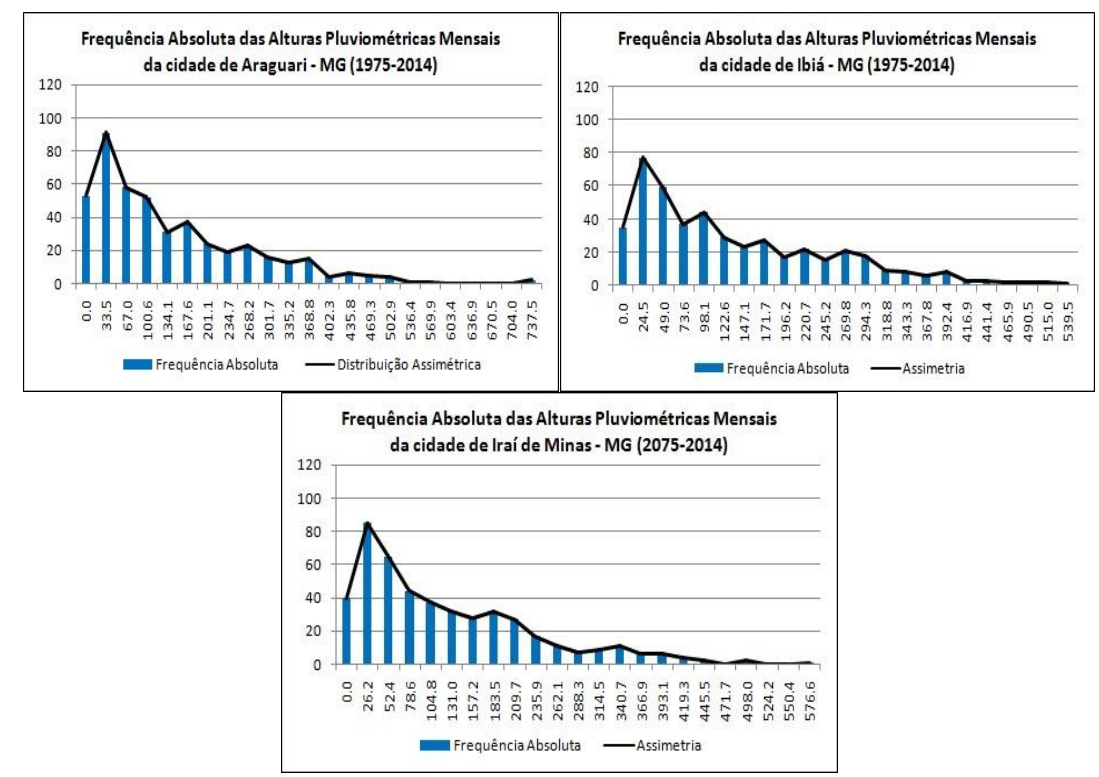



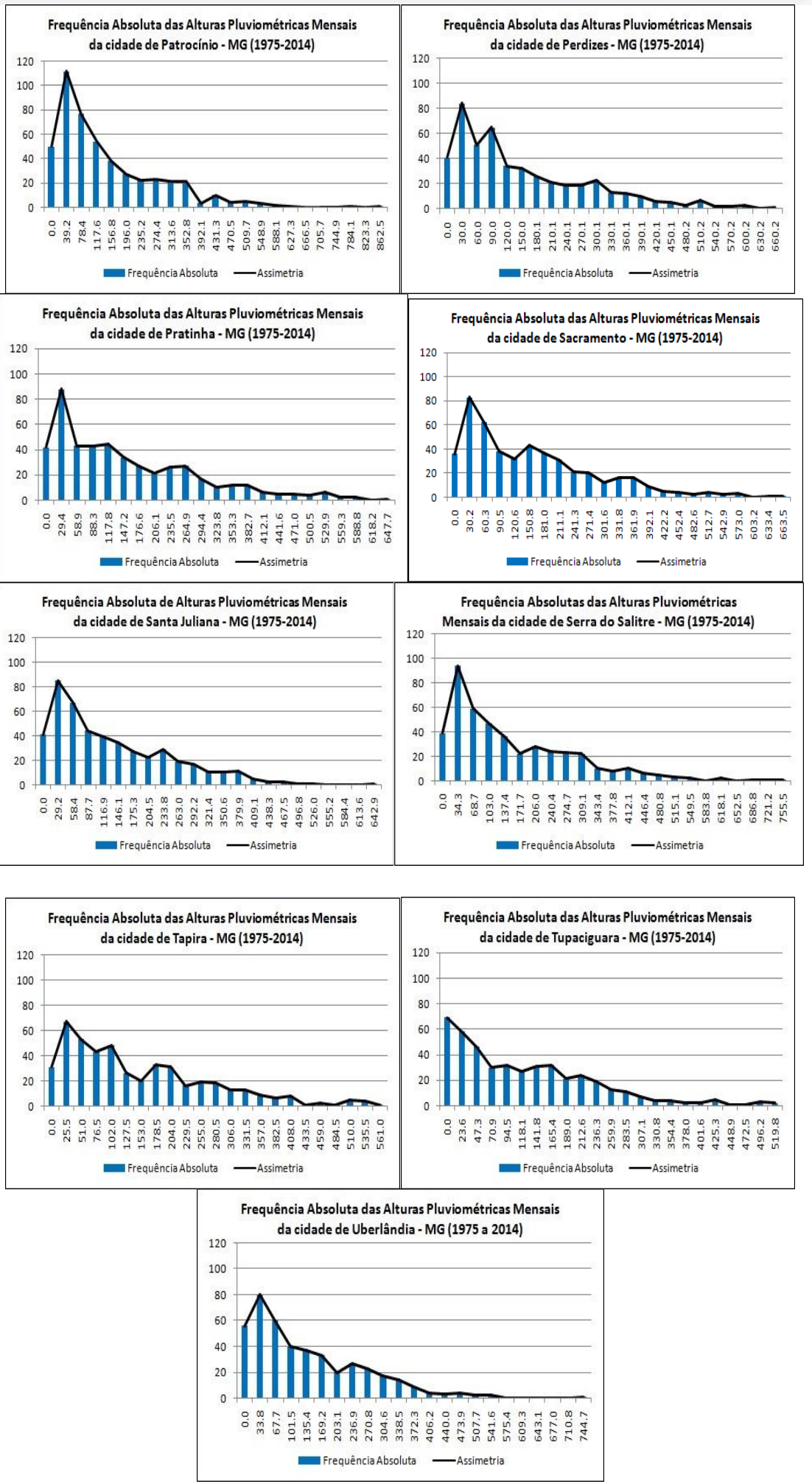

Figura 3 - Frequências Absolutas das precipitaçõesmensais acumuladas dos postos pluviométricos localizados na área de estudo (1975-2014).

Fonte dos dados: HidroWeb/ANA (2017).

Organização: MACIEL, S. A. (2017). 
A tabela II, traz os valores de Coeficiente de Assimetria (AS), sua classificação e seu respectivo grau de assimetria para cada posto da área de estudo.

Tabela II - Coeficiente de Assimetria dos postos pluviométricos localizados na área de estudo

\begin{tabular}{|c|c|c|c|c|}
\hline \multicolumn{5}{|c|}{ Coeficiente de Assimetria na bacia hidrográfica do Rio Araguari - (MG) } \\
\hline $\begin{array}{l}\text { Código } \\
\text { do Posto }\end{array}$ & $\begin{array}{l}\text { Município } \\
\text { de } \\
\text { Instalação }\end{array}$ & $\begin{array}{l}\text { Coeficiente de } \\
\text { Assimetria } \\
\text { (AS) }\end{array}$ & Classificação & $\begin{array}{c}\text { Grau } \\
\text { de } \\
\text { Assimetria }\end{array}$ \\
\hline 1848010 & Araguari & 0,96 & $\begin{array}{l}\text { Distribuição Assimétrica Positiva ou } \\
\text { à Direita } \\
\text { Distribuição Assimétrica Positiva ou }\end{array}$ & $\begin{array}{l}\text { Assimetria } \\
\text { Moderada } \\
\text { Assimetria }\end{array}$ \\
\hline 1946004 & $\begin{array}{l}\text { Ibiá } \\
\text { Iraí de }\end{array}$ & 1,05 & $\begin{array}{c}\text { à Direita } \\
\text { Distribuição Assimétrica Positiva ou }\end{array}$ & $\begin{array}{c}\text { Forte } \\
\text { Assimetria }\end{array}$ \\
\hline 1847010 & Minas & 1,02 & $\begin{array}{c}\text { à Direita } \\
\text { Distribuicão Assimétrica Positiva ou }\end{array}$ & $\begin{array}{c}\text { Forte } \\
\text { Assimetria }\end{array}$ \\
\hline 1846002 & Patrocínio & $\mathbf{0 , 9 2}$ & $\begin{array}{c}\text { à Direita } \\
\text { Distribuição Assimétrica Positiva ou }\end{array}$ & $\begin{array}{r}\text { Moderada } \\
\text { Assimetria }\end{array}$ \\
\hline 1947007 & Perdizes & $\mathbf{1 , 0 0}$ & $\begin{array}{c}\text { à Direita } \\
\text { Distribuição Assimétrica Positiva ou }\end{array}$ & $\begin{array}{c}\text { Forte } \\
\text { Assimetria }\end{array}$ \\
\hline 1946010 & Pratinha & 1,04 & $\begin{array}{c}\text { à Direita } \\
\text { Distribuição Assimétrica Positiva ou }\end{array}$ & $\begin{array}{c}\text { Forte } \\
\text { Assimetria }\end{array}$ \\
\hline 2047037 & $\begin{array}{l}\text { Sacramento } \\
\text { Santa }\end{array}$ & 1,07 & $\begin{array}{c}\text { à Direita } \\
\text { Distribuição Assimétrica Positiva ou }\end{array}$ & $\begin{array}{c}\text { Forte } \\
\text { Assimetria }\end{array}$ \\
\hline 1947001 & $\begin{array}{l}\text { Juliana } \\
\text { Serra do }\end{array}$ & 1,04 & $\begin{array}{c}\text { à Direita } \\
\text { Distribuição Assimétrica Positiva ou }\end{array}$ & $\begin{array}{c}\text { Forte } \\
\text { Assimetria }\end{array}$ \\
\hline 1946008 & Salitre & $\mathbf{0 , 9 8}$ & $\begin{array}{c}\text { à Direita } \\
\text { Distribuição Assimétrica Positiva ou }\end{array}$ & $\begin{array}{r}\text { Moderada } \\
\text { Assimetria }\end{array}$ \\
\hline 1946011 & Tapira & 1,10 & $\begin{array}{c}\text { à Direita } \\
\text { Distribuição Assimétrica Positiva ou }\end{array}$ & $\begin{array}{c}\text { Forte } \\
\text { Assimetria }\end{array}$ \\
\hline 1848004 & Tupaciguara & 1,02 & $\begin{array}{c}\text { à Direita } \\
\text { Distribuição Assimétrica Positiva ou }\end{array}$ & $\begin{array}{c}\text { Forte } \\
\text { Assimetria }\end{array}$ \\
\hline 1948006 & Uberlândia & 0,99 & à Direita & Moderada \\
\hline
\end{tabular}

Fonte dos dados: HidroWeb/ANA (2017)

Organização: MACIEL, S. A. (2017)

Em todas as estações pluviométricas da bacia hidrográfica do Rio Araguari - (MG) os coeficientes de assimetria foram maior que 0 , indicando uma distribuição assimétrica positiva ou à direita. Sendo que, Araguari apresentou o menor Coeficiente de Assimetria $(0,96)$ e Tapira o maior Coeficiente de Assimetria (1,10). Dos 12 postos de estudo, 4 deles (Araguari, Patrocínio, Serra do Salitre e Uberlândia), apresentaram assimetria moderada, ou seja, coeficientes que estiveram neste intervalo de $0,15<$ AS < 1. Já, 8 deles (Ibiá, Iraí de Minas, Perdizes, Pratinha, Sacramento, Santa Juliana, Tapira e Tupaciguara) demonstraram assimetria forte, ou seja, coeficientes situados nesta condição AS $\geq 1$.

Por fim, a tabela III corrobora com os resultados por meio dos valores do Coeficiente de Curtose (C) e o tipo de curva atribuido a cada posto da bacia.

Tabela II - Coeficiente de Curtose dos postos pluviométricos localizados na área de estudo

\begin{tabular}{cccc}
\hline & \multicolumn{4}{c}{ Coeficiente de Curtose na bacia hidrográfica do Rio Araguari - (MG) } \\
\hline Código & Município de Instalação & Coeficiente de Curtose (C) & Tipo de Curva \\
\hline 1848010 & Araguari & 0,277 & Platicúrtica \\
1946004 & Ibiá & 0,316 & Platicúrtica
\end{tabular}


XVII Simpósio Brasileiro

de Geografia Fisica Aplicada

I Congresso Nacional

de Geografia Física

OS DESAFIOS DA GEOGRAFIA FÍSICA NA FRONTEIRA DO CONHECIMENTO

Instituto de Geociências - Unicamp

Campinas - SP

28 de Junho à 02 de Julho de 2017

\begin{tabular}{lccc}
1847010 & Iraí de Minas & $\mathbf{0 , 2 9 8}$ & \multicolumn{1}{c}{ Platicúrtica } \\
1846002 & Patrocínio & $\mathbf{0 , 2 2 2}$ & Leptocúrtica \\
1947007 & Perdizes & $\mathbf{0 , 3 1 6}$ & Platicúrtica \\
1946010 & Pratinha & $\mathbf{0 , 3 1 7}$ & Platicúrtica \\
2047037 & Sacramento & $\mathbf{0 , 2 5 1}$ & Leptocúrtica \\
1947001 & Santa Juliana & $\mathbf{0 , 3 3 0}$ & Platicúrtica \\
1946008 & Serra do Salitre & $\mathbf{0 , 3 3 0}$ & Platicúrtica \\
1946011 & Tapira & $\mathbf{0 , 2 5 1}$ & Leptocúrtica \\
1848004 & Tupaciguara & $\mathbf{0 , 3 5 9}$ & Platicúrtica \\
1948006 & Uberlândia & $\mathbf{0 , 2 7 7}$ & Platicúrtica
\end{tabular}

Fonte dos dados: HidroWeb/ANA (2017)

Organização: MACIEL, S. A. (2017)

Dentre todos os postos pluviométricos, Sacramento e Serra do Salitre apresentaram os menores valores de Coeficiente de Curtose (0,251 ambos) e Tupaciguara o maior valor de Coeficiente de Curtose $(0,359)$. Portanto, dos 12 postos, 3 deles (Patrocínio, Sacramento e Tapira) apresentaram curva Leptocúrtica, ou seja, C < 0,263. E 9 postos (Araguari, Ibiá, Iraí de Minas, Perdizes, Pratinha, Santa Juliana, Serra do Salitre, Tupaciguara e Uberlândia) corresponderam a curva Platicúrtica, ou seja, C > 0,263 .

\section{Considerações Finais}

Diante dos métodos aplicados neste trabalho e que envolveram as etapas para determinação das frequências absolutas e coeficientes de assimetria e curtose, verificou-se a importância do entendimento a cerca das distribuições de frequência das chuvas, pois a partir dele pode-se estabelecer os melhores procedimentos estatísticos a serem utilizados na análise do comportamento desta variável.

A aplicação dos coeficientes de Assimetria e Curtose neste estudo se mostrou satisfatória, uma vez que tais valores complementam as medidas de posição e dispersão, auxiliando na compreensão mais detalhada das distribuições de frequências de dados pluviométricos e técnicas de construções de classes.

Dentre todos os postos (12) selecionados na bacia hidrográfica, 33,3\% ou 4 deles foram classificados como Distribuição Assimétrica Positiva Moderada e 66,7\% ou 8 deles foram categorizados como Distribuição Assimétrica Positiva Forte. Em termos de percentagens também, 25\% ou 3 deles apresentaram curva Leptocúrtica e 75\% ou 9 deles expressaram curva Platicúrtica.

Espera-se que trabalhos como este possam subsidiar outras pesquisas que partam do princípio do entendimento a cerca da variabilidade existente nas distribuições de frequência pluviométrica com referência na curva ou forma das sequências temporais. 


\section{Agradecimentos}

À Universidade Federal de Uberlândia (UFU), ao Instituto de Geografia (IG) e à FAPEMIG (Fundação de Amparo à Pesquisa do Estado de Minas Gerais) pelo apoio e financiamento na participação do evento.

\section{Bibliografia}

AYOADE, J. O..Introdução a climatologia para os trópicos. 4. ed. Rio de Janeiro: Bertrand Brasil, 1996. 332 p.

CHRISTOFOLETTI, A. L. H.. Procedimentos de análise utilizados no estudo da precipitação. Geociências, São Paulo, v.11, n. 6, p. 75-98, 1992.

GOMES, Ariel Ortiz. Modelagem estatística da precipitação: estudo de caso da bacia do rio Taguarizinho / MS. 2011. 63 f. Dissertação (Mestrado) - Curso de Tecnologias Ambientais, Universidade Federal de Mato Grosso do Sul, Campo Grande, 2011. Disponível em: <http://repositorio.cbc.ufms.br:8080/jspui/handle/123456789/2185>. Acesso em: 17 fev. 2017.

HOLTZ, Antonio Carlos Tatit. Precipitação. In: PINTO, Nelson L. de Sousa et al. Hidrologia Básica. São Paulo: Edgard Blücher, 1976. Cap. 2. p. 27-35.

MEDRI, Waldir. Análise exploratória de dados. 2011. 82 f. Curso de Estatística, Universidade Estadual de Londrina, $\quad$ Londrina, 2011. $\quad$ Disponível $\quad$ em: <http://www.uel.br/pos/estatisticaeducacao/textos_didaticos/especializacao_estatistica.pdf $>$. Acesso em: 16 fev. 03 .

MELLO, Yara Rúbia de; OLIVEIRA, Therezinha Maria Novais de. Análise Estatística e Geoestatística da Precipitação Média para o Município de Joinville (SC). Revista Brasileira de Meteorologia, São Paulo, v. 31, n. 2, p.229-239, 2016. Disponível em: <http://www.scielo.br/pdf/rbmet/v31n2/0102-7786-rbmet-31-020229.pdf>. Acesso em: 14 fev. 2017.

SILVA, Samuel de A.; LIMA, Julião S. de S.; BOTTEGA, Eduardo L.. Variabilidade espacial da precipitação pluviométrica para o Estado do Espírito Santo utilizando métodos multivariados. Revista Brasileira de Ciências Agrárias, Recife, $\quad$ v. $\quad 6, \quad$ n. $\quad 4, \quad$ p.703-709, 2011. Disponível em: <http://agraria.pro.br/sistema/index.php?journal=agraria\&page=article\&op=view\&path;[]=v6i4a834\&path;[]=10 32>. Acesso em: 14 fev. 2017.

SOCCOL, Olívio J.; CARDOSO, Célio O.; MIQUELLUTI, David J.. Análise da precipitação mensal provável para o município de Lages, SC. Revista Brasileira de Engenharia Agrícola e Ambiental, Campina Grande, v. 14, n. 6, p.569-574, 2010. Disponível em: 〈http://www.scielo.br/pdf/rbeaa/v14n6/a01v14n6.pdf〉. Acesso em: 15 fev. 2017.

VIEIRA, Sidney R.; CARMARGO, Marcelo B. P. de; SIQUEIRA, Glécio M.. Análise espacial da temperatura e da precipitação média anual no estado de São Paulo (Brasil). Revista Brasileira de Engenharia de $\begin{array}{lllllll}\text { Biossistemas, Tupã, } & \text { v. } \quad 3, \quad \text { n. } \quad 2, \quad \text { p.87-97, } 2009 . & \text { Disponível em: }\end{array}$ <http://seer.tupa.unesp.br/index.php/BIOENG/article/view/59/60>. Acesso em: 14 fev. 2017. 